\title{
An investigation of ACE as a risk factor for dementia and cognitive decline in the general population
}

\author{
A G Yip, C Brayne, D Easton, D C Rubinsztein, the Medical Research Council Cognitive \\ Function and Ageing Study (MRC CFAS)*
}

J Med Genet 2002;39:403-406

D ementia is the most common neurodegenerative condition affecting older people. It is estimated that around 550000 subjects aged 65 years and over in England and Wales suffer from dementia of mild or greater severity. ${ }^{1}$ Prevalence increases exponentially with age, from around $1 \%$ of 65 year olds to approximately $30 \%$ of people aged 85 years and older. ${ }^{2}$ Most cases (60-70\%) of incident dementia have clinical diagnoses of Alzheimer's disease, while 15-20\% are accounted for by vascular dementia (VaD). ${ }^{3}$ However, Alzheimer-type and vascular pathology frequently occur in the same person, the neuropathological features associated with $\mathrm{AD}$ and VaD are present in many cognitively intact people, and some demented subjects do not have the neuropathological hallmarks of $\mathrm{AD}$ or VaD. ${ }^{4}$

Alzheimer's disease risk is unequivocally associated with polymorphisms in the apolipoprotein E (APOE) gene. However, $A P O E$ accounts for around half of the genetic risk for AD. Recent data suggest that the angiotensin-I converting enzyme or ACE gene (chromosome 17q23) may also be involved in genetic susceptibility to AD. ${ }^{67}$ ACE (OMIM * $106180^{5}$ ) is a dipeptidyl carboxypeptidase that plays an important role in blood pressure regulation and electrolyte balance by hydrolysing angiotensin-I into angiotensin-II, a potent vasopressor and aldosterone stimulating peptide, and inactivating bradykinin, a potent vasodilator. An insertion (I)/deletion (D) polymorphism situated in intron 16 of the gene accounts for $50 \%$ of the interperson variability of plasma ACE concentration, and its links with myocardial infarction and other ischaemic heart disease and longevity have been studied extensively. ${ }^{5}$ There are few published studies on the association between the ACE I/D polymorphism and AD risk: Kehoe et al ${ }^{6}$ reported increased risk for $\mathrm{AD}$ among I allele carriers; however, this result was not uniformly replicated in subsequent studies. Results from our pooled analysis suggest a slightly increased risk (odds ratio $1.2,95 \%$ confidence interval 1.1 to 1.3 ) for $\mathrm{AD}$ among I allele carriers. ${ }^{7}$

We believe that the public are primarily concerned about their risk for dementia in general, in addition to the specific risks of AD or VaD. Similarly, from a public health perspective, it is crucial to understand how the ACE D/I polymorphism impacts on dementia in the general population as well as among narrowly diagnosed, selected, patient groups.

Thus we have investigated the effects of ACE on dementia risk, cognitive function, and rates of cognitive decline (as measured by serial Mini Mental State Examination scores) in elderly populations across England and Wales drawn from a multicentre population based longitudinal study of ageing and cognitive and functional status (the MRC Cognitive Function and Ageing Study).

\section{METHODS}

The MRC Cognitive Function and Ageing Study is a multicentre prospective study into the functional and cognitive status of the elderly in England and Wales. The centres used for the present analyses are set in two rural (Cambridgeshire and Gwynedd) and two urban (Nottingham and Newcastle) locations. A detailed description of the design of the study has been published elsewhere. ${ }^{1}$ Briefly, a two phase sampling design was used, where stratification for probability sampling for assessment was based on age and performance on dementia screening tests (automated geriatric examination for computer assisted taxonomy (AGECAT) organicity items $^{8}$ and the Mini Mental Status Examination ${ }^{9}$ ). A total of 2034 subjects out of 10264 screened were sampled for assessment in this way. The assessed respondents then underwent two further follow up interviews to ascertain incident cases. Those not sampled in the first (prevalence) wave were screened during the second wave (two years later), and were selected for assessment using the same probability sampling scheme (5618 screened, 1151 assessed). At the third wave (approximately six years into the study), a combined screen and assessment was completed on 1730 of this cohort who were still alive, accessible, and who had consented to remain in the study. Of this number, 1070 consented to give a blood sample or buccal swab for genetic studies.

Respondents were assessed by trained interviewers from professions allied to medicine. The assessment interview provides information processed by the computer algorithm AGECAT, ${ }^{8}$ which generates a diagnosis of dementia (as well as affective and anxiety disorders) on the basis of criteria compatible with those of the International Classification of Diseases, 9th revision (ICD-9 $)^{10}$ and the Diagnostic and Statistical Manual (DSM-IIIR). ${ }^{11}$ Dementia is diagnosed if a person has an AGECAT organicity rating of $\mathrm{O} 3$ or above, which is highly correlated with clinical assessment of dementia status. ${ }^{8}$

Cases comprised all blood/saliva contributing participants who had been assigned an AGECAT organicity level of O3 or higher at any point during the three assessment waves and an MMSE score of 21 or less at the third assessment wave. The control group comprised subjects with AGECAT organicity level below $\mathrm{O} 3$ and an MMSE score of 26 or greater at the third assessment wave. Cases and controls were drawn from the 913 subjects who had both $A P O E$ (a known genetic risk factor for dementia) and $A C E$ genotypes.

There were 118 cases $($ male $=33$ : median age at wave $3=81$, 25 th centile $=78,75$ th centile $=85 ;$ female $=85$ : median age $=86,25$ th centile $=82,75$ th centile $=89$ ) and 433 controls $($ male $=208$ : median age at wave $3=75,25$ th centile $=72,75$ th 


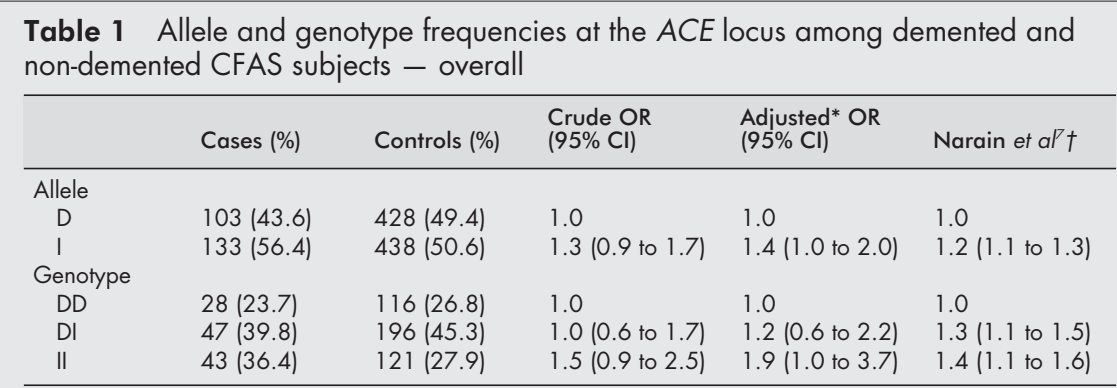

*Adjusted for age, sex, years in full time education, and $A P O E \in 4$ carrier status (for genotype). †Pooled estimate OR $(95 \% \mathrm{CI})$ for Alzheimer's disease.

centile $=79.5$; female $=225$ : median age at wave $3=76,25$ th centile $=72,75$ th centile $=81$ ).

APOE genotyping was performed as described by Wenham et $a l^{12}$ and ACE genotypes were determined using the method described by Evans et al. ${ }^{13}$ All genotyping was carried out by the first author $(\mathrm{AY})$, and genotype assignments double checked for accuracy by the corresponding author (DCR). Samples with ambiguous genotypes were rerun. Three genotyping attempts were performed for each sample, after which the APOE/ACE status of unsuccessfully typed samples were recorded as unknown/failed. APOE genotype was successfully determined in 1030 subjects and ACE genotype in 922. There are 913 subjects whose APOE and ACE genotypes are both known.
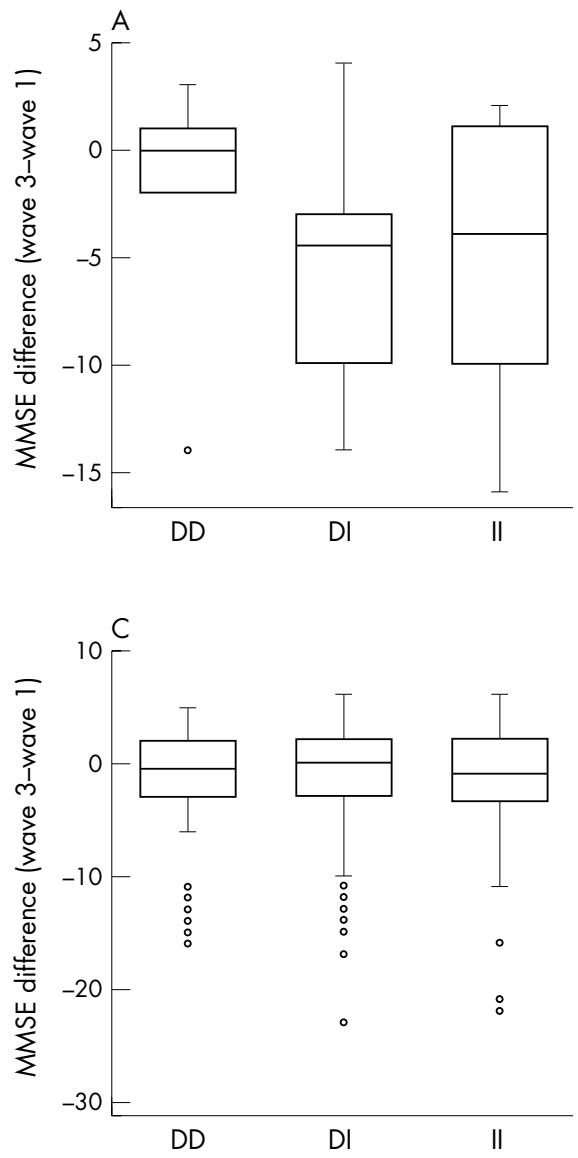

The association between ACE and dementia risk was examined by unconditional logistic regression. The analyses were conducted using third wave data only, to examine the cross sectional association between ACE and dementia risk, adjusting for age, sex, education, and $A P O E \in 4$ carrier status. Separate analyses for men and women, controlling for age and years in full time education were also carried out. The categorical variable ACE was classified by genotype: DD (reference group), DI, and II. Additionally, an odds ratio was calculated for allele I relative to D. This analysis assumes that the maternal and paternal alleles act multiplicatively on risk, so that, for example, the odds ratio associated with DI relative to DD is the product of the odds ratios of D and I (that is, the same as the odds ratio of I). Under this model the two alleles
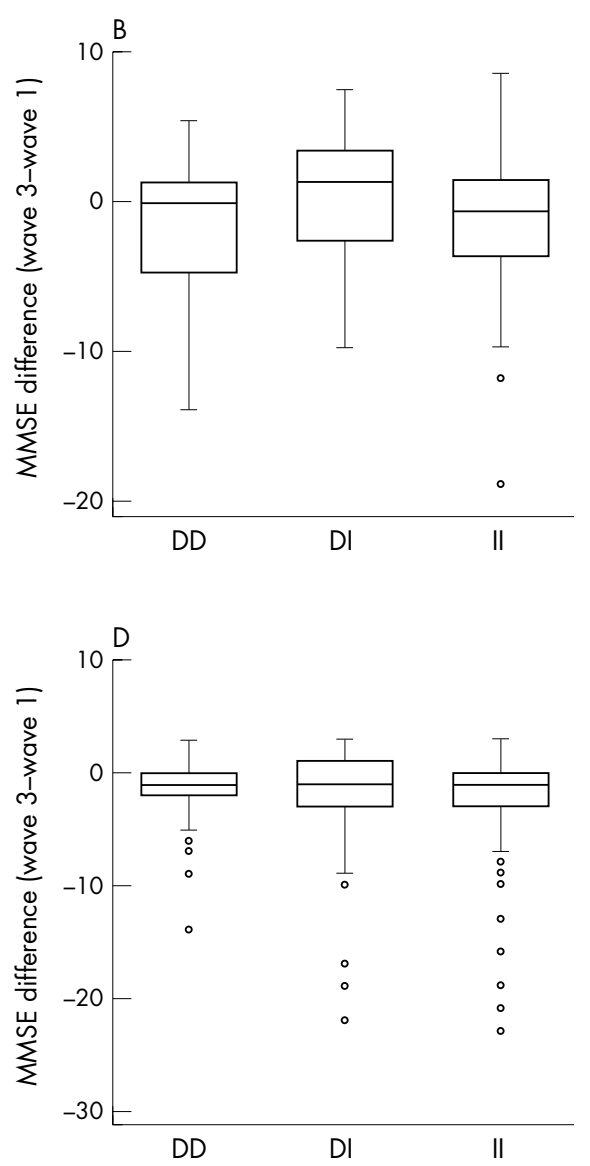

Figure 1 Distribution of MMSE difference (wave 3-wave 1) by ACE genotype, stratified by baseline MMSE score. (A) Baseline MMSE 17 or less: $\mathrm{DD}=5, \mathrm{DI}=15, \| l=10$. Kruskall-Wallis statistic $\left(\chi^{2}, 2 \mathrm{df}=1.8, \mathrm{p}=0.4\right)$. (B) Baseline MMSE 17-21: $\mathrm{DD}=24, \mathrm{Dl}=28, \|=27$. Kruskall-Wallis test $\left(\chi^{2}, 2 \mathrm{df}=1.1, p=0.6\right)$. (C) Baseline MMSE 22-25: $\mathrm{DD}=82, \mathrm{DI}=141, \|=83$. Kruskall-Wallis test $\left(\chi^{2}, 2 \mathrm{df}=1.6, p=0.4\right)$. (D) Baseline MMSE 26 and above: $\mathrm{DD}=118, \mathrm{Dl}=207, \|=129$. Kruskall-Wallis test $\left(\chi^{2}, 2 \mathrm{df}=0.2, \mathrm{p}=0.9\right)$. 

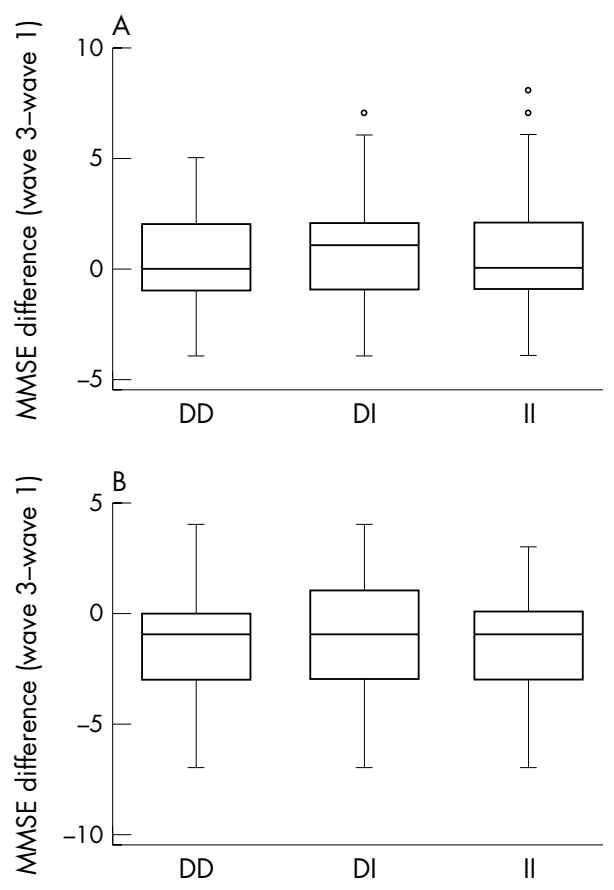

Figure 2 Distribution of MMSE difference by ACE genotype, stratified by wave 3 MMSE score. (A) MMSE wave 3 score 26-30. $\mathrm{DD}=111, \mathrm{Dl}=189, \|=119\left(\chi^{2}, 2 \mathrm{df}=0.513, \mathrm{p}=0.8\right)$. (B) MMSE wave 3 score $22-25$. $\mathrm{DD}=63, \mathrm{DI}=118, \|=65\left(\chi^{2}=0.6, \mathrm{p}=0.7\right)$.

from the same person can be analysed as if independent. Risk estimates based on genotype do not assume multiplicativity of risk..$^{14}$

Because of the negatively skewed distribution of MMSE, individual scores at wave 3 were log transformed $\left[\mathrm{MMSE}_{\text {transformed }}=\log _{\mathrm{e}}(31-\mathrm{MMSE})\right]$, pooled by genotype, and then compared using analysis of variance (cross adjusting for genotype, age, sex, and number of years in full time education). The mean transformed MMSE score of each ACE genotype was back transformed $\left[\mathrm{MMSE}_{\text {orignal }}=31\right.$-exp(mean $\left.\left.\mathrm{MMSE}_{\text {transformed }}\right)\right]$ to return mean scores to their original scale.

We used the difference in MMSE between score at baseline and at the third assessment wave as an index of cognitive decline. The Kruskall Wallis test was used to compare the distributions of MMSE differences across the different ACE genotypes; 869 subjects had MMSE wave 1 and MMSE wave 3 information.

\section{RESULTS}

Table 1 shows the distribution of ACE genotype and allele frequencies for cases and controls, as well as crude and adjusted (for age, sex, years in full time education, and APOE4 carrier status) odds ratios. The distribution of ACE genotypes and alleles tended towards an over-representation of the I allele among cases versus the controls and risk appeared to be higher in II than DI subjects. However, all the confidence intervals included 1.0. Sex specific data did not show differences in ACE genotype and allele effects on dementia risk (data not shown).

The back transformed mean MMSE scores (see Methods) at wave 3 by ACE genotypes are: $\mathrm{DD}=25.5, \mathrm{DI}=25.6, \mathrm{II}=25.6$. MMSE scores at the third wave were not significantly different between ACE genotypes (F score $(2 \mathrm{df})=0.1, \mathrm{p}=0.9)$.

We were interested in the effects of ACE genotypes on the rate of cognitive decline, as measured by the difference in MMSE scores between the initial and third assessment waves, in our entire sample. In one set of analyses, we compared the distribution of MMSE differences across ACE genotypes in the

\section{Key points}

- The D/I polymorphism of the angiotensin-I converting enzyme (ACE) gene has been postulated to be associated with Alzheimer's disease risk. It is crucial to understand how this genetic factor impacts on dementia in the general population, as well as among narrowly diagnosed, selected, patient groups.

- The ACE D/I polymorphism may have only very modest effects on dementia risk in the general population and appears not to affect cognitive decline in the elderly population.

- A study with larger sample sizes is needed to enable exclusion of any small effects at this locus on dementia in the general population.

sample separated into four subgroups based on people's MMSE scores at the prevalence wave (namely, 0 to 17,18 to 21,22 to 25 , and 26 to 30 ). We adopted this strategy, since a given difference in MMSE scores over time would have a different meaning depending on the starting MMSE score. In another analysis, we looked at the distribution of MMSE difference across the different ACE genotypes among subjects scoring between 22 and 25 and between 26 and 30 on the MMSE at wave 3. This strategy was used to see whether ACE genotypes affected the rate of decline in people who were ostensibly non-demented throughout the study. In other words, we wanted to test if ACE affected the rate of cognitive decline before the onset of dementia.

Fig 1 shows the distribution of the difference in MMSE score between assessment waves 1 and 3 across ACE genotypes, according to baseline MMSE level. Fig 2 shows the MMSE difference across ACE genotypes among nondemented subjects at wave 3 who scored between 22 and 25 and 26 and over on the MMSE. There are no discernible differences across ACE genotypes when the sample was analysed using either of the strategies described above. Furthermore, ACE genotypes did not have any effect on change in MMSE score when the entire sample was analysed without any stratification (data not shown).

\section{DISCUSSION}

The aim of this study was to investigate the impact of ACE on dementia in the general population. This is in contrast with most published studies (both clinic/necropsy based and population based), which have looked into the impact of ACE on $\mathrm{AD}$ risk specifically, and have tended to use younger samples which may not reflect the population in which dementia occurs most frequently.

The MRC CFAS study uses AGECAT to diagnose dementia. It is a robust algorithm, with a high overall index of agreement $(\kappa=0.78)$ with psychiatrists' diagnoses in both the community and geriatric hospital settings (and excellent agreement regarding diagnosis of organic disorders, $\kappa=0.82) .{ }^{15}{ }^{16}$ It is possible that the impact of ACE is greatest for AD pathology and may have less importance for the other processes that contribute to dementia in the general population. While we failed to find a significant effect of the ACE I allele on dementia risk, the point estimates suggest a dose dependent increase in dementia risk consistent with results published in our meta-analysis.?

While ACE does not appear to have a major effect on dementia/AD risk in the general population, it warrants further investigation. Larger samples are required before one can exclude small effects that may be operating at this locus for dementia/AD; in order to achieve $80 \%$ power to detect an odds ratio of 1.2 , given $50 \%$ prevalence of the risk allele among 
non-cases, we would need just under 400 cases and controls. Robust confirmation of an AD risk gene is valuable, even if the effect is small, as it would contribute to our understanding of $\mathrm{AD}$ pathology and furthermore may suggest potential therapeutic strategies.

\section{ACKNOWLEDGEMENTS}

This work was supported by the Medical Research Council. The authors thank the MRC CFAS respondents and their relatives for their participation in this long running project, and the MRC CFAS Internal Cambridge Advisory Group and Dr David Clayton for comments. AY is grateful to the Cambridge Overseas Trust and the European Dana for the Brain for funding. DCR is a Glaxo Wellcome Research Fellow.

\section{Authors' affiliations}

A G Yip, C Brayne, Department of Public Health and Primary Care, University Forvie Site, Robinson Way, Cambridge CB2 2SR, UK

A G Yip, D C Rubinsztein, Department of Medical Genetics, Cambridge Institute for Medical Research, Wellcome/MRC Building, Addenbrooke's Hospital, Hills Road, Cambridge CB2 2XY, UK

D Easton, CRC Genetic Epidemiology Unit, Cambridge University Department of Public Health and Primary Care, Strangeways Research Laboratory, Worts' Causeway, Cambridge CB1 8RN, UK

Correspondence to: Dr D C Rubinsztein, Department of Medical Genetics, Cambridge Institute for Medical Research, Wellcome/MRC Building, Addenbrooke's Hospital, Hills Road, Cambridge CB2 2XY, UK dcr1000@cus.cam.ac.uk

\section{REFERENCES}

1 The Medical Research Council Cognitive Function and Ageing Study (MRC CFAS). Cognitive function and dementia in six areas of England and Wales: the distribution of MMSE and prevalence of GMS organicity level in the MRC CFA Study. Psychol Med 1998;28:319-35.

2 Lobo A, Launer LJ, Fratiglioni L, Andersen K, Di Carlo A, Breteler MM, Copeland JR, Dartigues JF, Jagger C, Martinez-Lage J, Soininen H, Hofman A. Prevalence of dementia and major subtypes in Europe: a collaborative study of population-based cohorts. Neurologic Diseases in the Elderly Research Group. Neurology 2000;54(11 suppl 5):S4-9.
3 Fratiglioni L, Launer L, Andersen K, Breteler MM, Copeland JR Dartigues JF, Lobo A, Martinez-Lage J, Soininen H, Hofman A. Incidence of dementia and major subtypes in Europe: a collaborative study of population-based cohorts. Neurologic Diseases in the Elderly Research Group. Neurology 2000;54(11 suppl 5):S10-15

4 Neuropathology Group of the Medical Research Council Cognitive Function and Ageing Study (MRC CFAS). Pathological correlates of late-onset dementia in a multicentre, community-based population in England and Wales. Lancet 2001;357:169-75.

5 Online Mendelian Inheritance in Man (OMIM). Angiotensin I converting enzyme. http://www3.ncbi.nlm.nih.gov/htbin-post/Omim/ dispmim? 106180 2001; accessed 21 September 2001.

6 Kehoe PG, Russ C, Mcllory S, Williams H, Holmans P, Holmes C, Liolitsa D, Vahidassr D, Powell J, McGleenon B, Liddell M, Plomin R, Dynan K, Williams N, Neal J, Cairns NJ, Wilcock G, Passmore P, Lovestone S, Williams J, Owen M. Variation in DCP1, encoding ACE, is associated with susceptibility to Alzheimer disease. Nat Genet 1999;21:71-2.

7 Narain Y, Yip A, Murphy T, Brayne C, Easton D, Evans JG, Xuereb J, Cairns N, Esiri MM, Furlong RA, Rubinsztein DC. The ACE gene and Alzheimer's disease susceptibility. J Med Genet 2000;37:695-7.

8 Copeland JRM, Dewey ME, Griffiths-Jones HM. Computerised psychiatric diagnostic system and case nomenclature for elderly subjects: GMS and AGECAT. Psychol Med 1986:16:89-99.

9 Folstein MF, Folstein SE, Hugh PR. Mini-mental state: a practical method for grading the cognitive state of patients for the clinician. J Psychiatr Res 1975;313:1419-20.

10 World Health Organization. Tenth Revision of the International Classification of Diseases. Geneva: World Health Organization, 1992.

11 American Psychiatric Association. Diagnostic and statistical manual, edition III. Revised. Washington, DC: American Psychiatric Association, 1987.

12 Wenham PR, Price WH, Blundell G. Apolipoprotein E genotyping by one-stage PCR. Lancet 1991;337:1158-9.

13 Evans AE, Poirier O, Kee F. Polymorphisms of the angiotensin-converting enzyme gene in subjects who die from coronary heart disease. Q J Med 1994:87:211-14

14 Rubinsztein DC, Easton DF. Apolipoprotein E genetic variation and Alzheimer's disease. a meta-analysis. Dement Geriatr Cogn Disord 1999;10:199-209

15 Collinghan G, Macdonald A, Herzberg J, Philpot M, Lindesay J. An evaluation of the multidisciplinary approach to psychiatric diagnosis in elderly people. BM 1993:306:821-4

16 Ames D, Flynn E, Tuckwell V, Harrigan S. Diagnosis of psychiatric disorder in elderly general and geriatric hospital patients: AGECAT and DSM-IIIR compared. Int J Geriatr Psychiatry 1994;9:627-33.

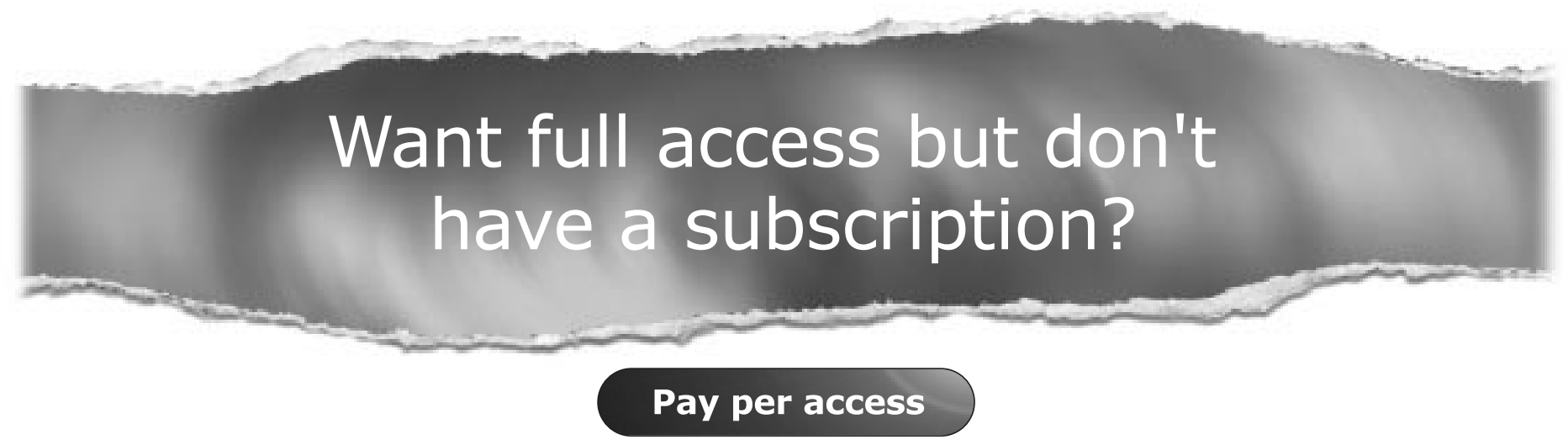

For just US\$25 you can have instant access to the whole website for 30 days. During this time you will be able to access the full text for all issues (including supplements) available. You will also be able to download and print any relevant pdf files for personal use, and take advantage of all the special features Journal of Medical Genetics online has to offer.

\section{www.jmedgenet.com}

